\title{
EVALUACION DEL COMPONENTE CENTRAL Y PERIFERICO DE FATIGA MUSCULAR EN PACIENTES NEUROPATICOS Y MIOPATICOS
}

\author{
L. G. COHEN \\ MARCELA E. PANIZZA \\ OLGA P. SANZ \\ CARLOS G. SCHUTZ \\ R. $R E Y$
}

La metodología descripta para evaluar fatiga muscular 2 fue aplicada a dos grupos de pacientes con procesos denervatorios crónicos y daño muscular primitivo.

\section{MATERIAL Y METODOS}

Se estudiaron 10 pacientes, 6 hombres y 4 mujeres. En algunos casos se realizó el estudio unilateralmente $y$ en otros bilateralmente. 7 padecian procesos denervatorios de grado moderado y en recuperación (2 polineuropatías, 2 esclerosis luteral amiotrófica, 2 radiculopatías) en tanto que otros 3 estaban afectados por daño muscular primitivo de tipo distrófico ( 1 enfermedad de Steinert, 1 enfermedad de Duchenne y 1 miopatía no clasificada). En pacientes denervados agudos o severos no se pudo lograr el mantenimiento del esfuerzo por un lapso suficiente para ser valorado. Controles - Se utilizaron como controles 13 sujetos, 10 hombres y 3 mujeres sanos y voluntários 2. Métodos - Los detalhes de la metodología empleada, así como el tratamiento estadístico de los resultados se dan en otro trabajo 2.

\section{RESULTADOS}

1. Onda $M$ máxima - La amplitud de la onda $M$ máxima obtenida al cabo del esfuerzo disminuyó al $99.12 \pm 12.75 \%$ de su valor inicial en pacientes neuropáticos y al $88.96 \pm 11.62 \%$ en los pacientes con dano muscular primitivo, sin que existieran en relación a los valores controles diferencias significativas (controles 92.59土11.37\%).

2. Frecuencia de los potenciales positivos y negativos que integran el electromiograma (EMG) - a. Grupo de pacientes denervados: la frecuencia de los potenciales positivos y negativos disminuyó al $66.03 \pm 57 \%$ y $89.35 \pm 32.56 \%$ respectivamente en tanto en los controles dicha frecuencia disminuyó al $85.05 \pm 45.24 \%$ y $96.14 \pm 58.16 \%$ respectivamente; tan solo la frecuencia de los potenciales positivos mostró una disminución discretamente significativa $(p<0.05)$ en estos pacientes. $b$. Grupo de pacientes con dano muscular primitivo: la frecuencia de los potenciales positivos disminuyó al $61.67 \pm 40.07 \%$ en tanto la de los negativos lo hizo al $65 \pm 49.50 \%$; esto no constituyó

Sección de Electroneurofisiología Clínica, Hospital Ramos Mejía. Buenos Aires, Argentina. 
una diferencia estadisticamente significativa con los valores controles (controles $85.05 \pm 45.24 \%$ y $96.14 \pm 58.16 \%$ ); se observó la misma tendencia a la disminución de frecuencia que en controles normales pero partiendo de valores inferiores a los mismos.

3. Durución de los potenciales positivos y negativos que integran el electromiograma (GMG) - a. Grupo de pacientes denervados: lá duración de los potenciales positivos (13.17 $\pm 10.36 \%$ ) mostró bruscos aumentos a los 2,4 y 8 minutos (coincidiendo a los 2 y 4 minutos con lo occurrido en pacientes miopáticos) en tanto que la duración de los potenciales negativos (154.12士90.26\%) mostró ese aumento sólo a los 2 y 8 minutos; la tendencia de las curvas fue ascendente tal cual se observó en los controles normales (controles $108.35 \pm 49.29 \%$ y $117.13 \pm 39.82 \%$ respectivamente) aunque sus cifras fueron mayores que las obtenidas en dicho grupo control; la diferencia entre ambos no fue estadisticamente significativa. $b$. Grupo de pacientes con daño muscular primitivo: la duración de los potenciales positivos (105.34 $\pm 49.29 \%$ y $117.13 \pm 39.82 \%$ respectivamente) al cabo de 6 minutos de esfuerzo, muestran la misma tendencia ascendente de
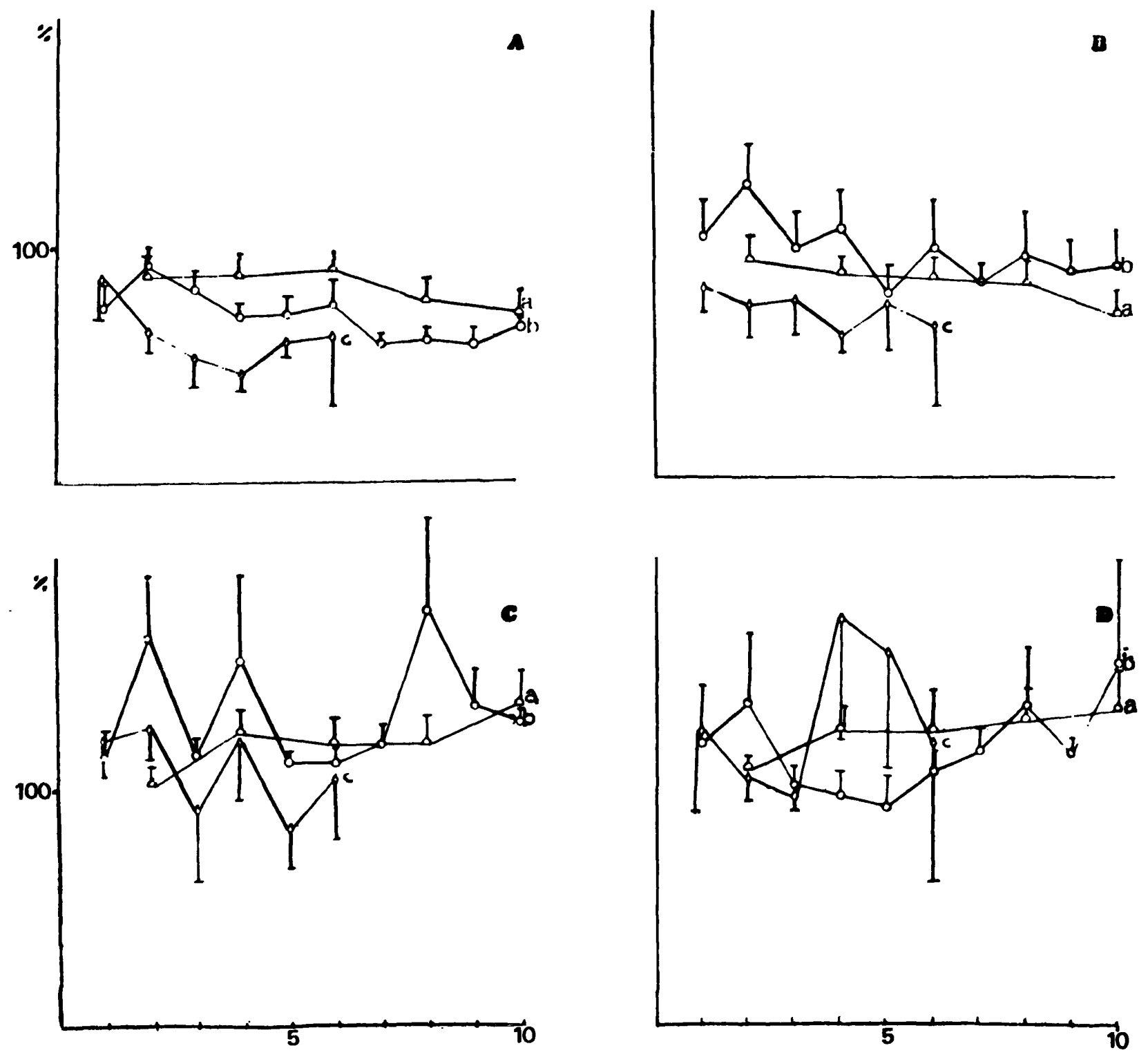

Fig. 1 - A. Frecuencia $(+)$ a: controles, b: pacientes denervados, $c$ : pacientes miopdticos. B. Frecuencia (-) a: controles, $b$ : pacientes denervados, $c$ : pacientes miopáticos. C. Duración (t) a: controles, b: pacientes denervados, c: pacientes miopáticos: D. Duracion (-) a: controles, b: pacientes denervados, $c$ : pacientes miopaticos. En abscisa tiempo de observacion en minutos. 
los sujetos controles (controles $108.35 \pm 49.29 \%$ y $117.13 \pm 39.82 \%$ respectivamente). Estas diferencias no fueron estadísticamente significativas. Se observó, asimismo, bruscos aumentos de la duración de los potenciales de estos pacientes a los 2 y 4 minutos retornando luego a valores inferiores.

\section{COMENTARIOS}

En el grupo de pacientes con daño muscular primitivo, los valores de frecuencia de batido de los potenciales de unidad motora, se mostraron por debajo de los controles desde el comienzo del experimnto. Este hecho puede ser interpretado a favor de la existencia de dos factores independientes, aunque tal vez concurrentes; el primero una posible menor capacidad de estos pacients para conseguir un ritmo de descarga de unidades motoras similar al de los normales y el otro, una eventual disminución del número de unidades motoras funcionantes en el músculo bajo exploración 1. De todas formas las unidades motoras que se mantuvieron activas, exhibieron un comportamiento paralelo al de los controles.

En el grupo de pacientes denervados, la frecuencia de batido de las unidades motoras no mostró diferencias mayores en relación a los valores controles; este hallazgo plantea la posibilidad teórica de que tratándose de pacientes que por su patología han perdido unidades motoras, las remanentes tengan capacidad de descarga lo suficiente aumentada como para realizar un trabajo muscular normal.

El comportamiento de las duraciones, varió a lo largo del estudio, observándose períodos de mayor sincronización de la descarga de potenciales, en algunos casos coincidentes en ambos grupos ( 2 y 4 minutos).

Consideradas globalmente las tendencias de las curvas de los pacientes denervados, parecen comportarse en forma semejante a los sujetos controles, a pesar de los altibajos referidos (períodos de mayor sincronización). Los miopáticos, en cambio, se encuentran por debajo del grupo control, hecho que podría deberse al menor número de unidades motoras en acción que muestran desde el comienzo y/o a la pérdida de fibras musculares individuales dentro de la unidad motora.

La onda $M$ mostró una mayor disminución de amplitud en pacientes miopáticos $(11 \%)$ que en denervados (1\%). Esto podría ser explicado por pérdida de unidades motoras reclutadas durante el esfuerzo o bien por pérdida de fibras musculares dentro de unidades motoras (por bloqueo en la transmisión neuromuscular) ${ }^{1}$. Lo observado, sugiere que: en el grupo de pacientes miopáticos además del componente central, que aparentemente subyace como mecanismo básicos de la fatiga muscular ${ }^{2}$, existen factores periféricos que contribuyen a su desarrollo, tales como la posible pérdida de unidades motoras funcionantes durante el esfuerzo sostenido o de fibras musculares aisladas por probable bloqueo temporario de la transmisión neuromuscular. En el grupo de enfermos con alteraciones denervatorias, la eventual pobreza de unidades motoras utilizadas durante el esfuerzo, lleva posiblemente a un reordenamiento 
central que se traduce en una mayor sincronización del número de unidades motoras batientes por unidad de tiempo.

\section{RESUMEN}

Utilizando un método de cuantificación del electromiograma, se investigaron parte de los mecanismos centrales y periféricos responsables de la fatiga muscular en enfermos crónicamente denervados y en pacientes con compromiso muscular primitivo. Se observó en los denervados que los mecanismos de fatiga muscular, no difieren mayormente de los observados en el grupo de sujetos sanos, por el contrario, en los miopáticos a más del componente central, existen otros de orden periférico, tales como la pérdida de unidades motoras funcionantes durante el esfuerzo y el bloqueo temporario de la transmisión neuromuscular, que contribuye al desarrollo de la fatiga.

\section{SUMMARY}

Muscular fatigue mechanisms in neuropathies and myopathies.

In a group of patients chronically denervated and other with primary muscle involvement a search was made for mechanisms involved in the muscular fatigue. It was found that there was no major differences between patients chronically denervated and controls, while in those others with primary muscle disorders a loss of functional motor units during effort and a transient impairment of neuromuscular transmission play a role together with the central events normally occuring in fatigue.

\section{REFERENCIAS}

1. MC COMAS, A. J.: SICA, R. E. P. \& UPTON, A. R. M. - Multiple muscle analysis of motor units in muscular dystrophy. Arch. Neurol. (Chicago) 30:249, 1974.

2. PANIZZA, Marcela E.; COHEN, L. G.; SANZ, Olga; REY, R.; SCHUTZ, C. Evaluación del factor central y periférico en fatiga muscular. Arq. Ne ro-Psiquiat., (São Paulo) $41: 241,1983$.

Sacción de Electroneurofisiología Clinica. Departamento de Neurologí. Hospital Ra. mos Mejia - Urquiza 609 - 1281 Buenos Aires, Argentina. 